Article

\title{
Every Single Specimen Counts: A New Docosia Winnertz (Diptera: Mycetophilidae) Species Described from a Singleton ${ }^{\dagger}$
}

\author{
Olavi Kurina *(D) and Heli Kirik
}

Citation: Kurina, O.; Kirik, H. Every Single Specimen Counts: A New Docosia Winnertz (Diptera:

Mycetophilidae) Species Described from a Singleton. Insects 2021, 12, 1069. https://doi.org/10.3390/ insects12121069

Academic Editors: Jan Ševčík, John K. Moulton and John Skartveit

Received: 9 November 2021

Accepted: 26 November 2021

Published: 29 November 2021

Publisher's Note: MDPI stays neutral with regard to jurisdictional claims in published maps and institutional affiliations.

Copyright: (c) 2021 by the authors. Licensee MDPI, Basel, Switzerland. This article is an open access article distributed under the terms and conditions of the Creative Commons Attribution (CC BY) license (https:// creativecommons.org/licenses/by/ $4.0 /)$.
Institute of Agricultural and Environmental Sciences, Estonian University of Life Sciences, Friedrich Reinhold Kreutzwaldi 5-D, 51006 Tartu, Estonia; heli.kirik@emu.ee

* Correspondence: olavi.kurina@emu.ee; Tel.: +372-5518020

† Nomenclatural Acts: LSID urn:lsid:zoobank.org:act: 6B1E8E5E-E745-44B4-A270-24973EF6C913.

Simple Summary: A new fungus gnat species has been described from a single specimen collected from Georgia (Sakartvelo). The new species, named after its occurrence in Caucasia as Docosia caucasica sp. n., is distinguished from congeners by the characters in male terminalia and a unique COI sequence. As a substantial proportion of species in ecological communities tend to be rare, about $20-30 \%$ of new insect taxa have been described from a singleton so far. Therefore, following high-quality standards when describing new species, particularly when dealing with minimalistic material, is crucial. As much as possible, using multiple sets of characters, like morphology and DNA sequencing, is encouraged.

Abstract: A new species-Docosia caucasica sp. n.- -has been described from material collected from the Lesser Caucasus Mountains in Georgia (Sakartvelo). The new species belongs to a group of Palaearctic species characterized by distinct posterolateral processes of gonocoxites and apically modified setae at the posteroventral margin of the gonocoxites medially. Within the group, D. caucasica sp. n. is most similar to D. landrocki Laštovka and Ševčík, 2006 in having a similar outline of the medial process of posteroventral margin of the gonocoxites and the gonostylus. There is also a marked difference within the partial cytochrome c oxidase subunit 1 gene (COI) sequence of $D$. caucasica sp. n. and other Docosia spp. available in public databases. As the new species is described from a single male specimen only, the adequacy and code compliance of that are discussed.

Keywords: COI; fungus gnats; Georgia; new species; singleton; taxonomy

\section{Introduction}

The genus Docosia Winnertz, 1864 of Mycetophilidae (Diptera) has gained considerable attention during the last few decades, based on both taxonomy as well as phylogeny. Having been classified traditionally within the subfamily Leiinae (or the tribus Leiini) by earlier authors [1-3], molecular studies have instead suggested it belongs to Gnoristinae [4,5]. However, in a recent synopsis of Leiinae by Oliveira and Amorim [6], a new subfamily Tetragoneurinae was established, consisting of four genera including Docosia. Intrageneric phylogeny based on five molecular markers has been proposed by Ševčík et al. [7]. Supplementing phylogenetic studies, a number of new species have been described during the last few decades from the Palaearctic (e.g. [8-13]), Nearctic [14,15], Oriental [16] and Neotropical $[17,18]$ realms. Altogether, 86 extant Docosia species have been described, including 62 from the Palaearctic realm [7]. Within Mycetophilidae, Docosia species are characterised by medium size, dark brown to black body colour, unmarked wings, details of wing venation and distinctive male terminalia with combs of small spines (retinacula) on cerci in particular [12,19]. The species level identification has so far relied mainly on the morphology of male terminalia. However, a considerable number of species has also DNA information uploaded to the public databases (https:/ / www.boldsystems.org/ (accessed 
on 2 November 2021); https: / / www.ncbi.nlm.nih.gov/genbank/ (accessed on 2 November 2021)) that enable and support species identification.

This communication is prompted by discovery of a new Docosia species from the material collected in Georgia (Sakartvelo). Because the new species is represented by a single male specimen only, the adequacy and code compliance of such a description is discussed.

\section{Materials and Methods}

The specimen was collected during 2019 in the Lesser Caucasus Mountains in Georgia by collaborators of ZFMK (Zoological Research Museum Alexander Koenig, Bonn, Germany) using a Malaise trap. The collecting locality was a small meadow (flat surface close to a small river) surrounded by riverine trees and forest. The material was collected into and thereafter preserved in ethyl alcohol. Fungus gnats were sorted out and identified, whereas one specimen was found to represent a new species and therefore subjected to detailed study.

One fore leg was detached and used for DNA sequencing. The sample was homogenized with Kontes Pellet Pestle (DWK Life Sciences GmbH, Mainz, Germany) and DNA extraction completed using DNeasy Blood and Tissue Kit (Qiagen, Hilden, Germany) according to manufacturer instructions. 710 bp partial cytochrome c oxidase subunit 1 (COI) sequences were replicated using the primer pair LCO1490 (5' - GGTCAACAAATCATAAA GATATTGG-3') and HCO2198 (5'- TAAACTTCAGGGTGACCAAAAAAT-3') [20], in a PCR mixture comprising $1 \mu \mathrm{L}$ template DNA, $12.5 \mu \mathrm{L}$ DreamTaq PCR Master Mix (Thermo Fisher Scientific, Waltham, MA, USA), $0.5 \mu \mathrm{L}$ of each primer and $10.5 \mu \mathrm{L}$ ddH2O. The PCR program included a $15 \mathrm{~min}$ initial denaturation stage at $94{ }^{\circ} \mathrm{C}$, followed by 60 cycles of $30 \mathrm{~s}$ denaturation at $94{ }^{\circ} \mathrm{C}, 30 \mathrm{~s}$ annealing at $44{ }^{\circ} \mathrm{C}$ and $30 \mathrm{~s}$ of synthesis at $72{ }^{\circ} \mathrm{C}$, finished by a $10 \mathrm{~min}$ syntheses stage at $72^{\circ} \mathrm{C}$. Sequencing was carried out with Sanger sequencing by the Institute of Genomics Core Facility (University of Tartu, Tartu, Estonia). Forward and reverse strands were integrated and primer sequences trimmed in BioEdit version 7.2.6.1 [21]. The resulting sequence was used to search the GenBank database (National Institutes of Health, Bethesda, USA) for closely related species. COI sequences of D. anatolica Ševčík in Ševčík et al., 2020, D. diutina Plassmann, 1996, D. fumosa Edwards, 1925, D. landrocki Laštovka and Ševčík, 2006, D. nigra Landrock, 1928, D. pannonica Laštovka and Ševčík, 2006, D. rohaceki Ševčík, 2006 were used for genetic analysis in MEGA11 [22]. The phylogenetic tree was constructed using the maximum likelihood method and the appropriate model for analysis was chosen with the Find Best DNA/Protein Models (ML) function in MEGA11. Furthermore, bootstrapping with 1000 iterations was employed to test phylogeny. However, values less than $75 \%$ were removed from the final figure for ease of viewing. All in all, the analysis involved eight sequences and a total of 658 nucleotide positions.

For a detailed study of the terminalia, they were detached and treated with ca. $10 \%$ warm potassium hydroxide $(\mathrm{KOH})$, followed by neutralization with acetic acid $\left(\mathrm{CH}_{3} \mathrm{COOH}\right)$ and washing with distilled water. Terminalia were studied in glycerine $\left(\mathrm{C}_{3} \mathrm{H}_{8} \mathrm{O}_{3}\right)$ and stored as glycerine preparations in small plastic vials attached to the rest of the specimen [23]. Illustrations of the terminalia were prepared using a U-DA drawing tube attached to a compound microscope Olympus CX31. The digital images of the habitus and terminalia were combined using the software LAS V.4.1.0. from multiple gradually focused images taken by a Leica DFC 450 camera attached to a Leica 205C stereomicroscope. Adobe Photoshop CS5 and Topaz Shapen Al were used for editing the figures, compiling the plates and improving sharpness. Morphological terminology follows Søli [19] and Ševčík et al. [7].

The holotype of the new species is deposited in the Zoological Research Museum Alexander Koenig, Bonn, Germany (ZFMK). Comparative material of Docosia landrocki and other species are deposited in the Institute of Agricultural and Environmental Sciences, Estonian University of Life Sciences, Tartu, Estonia (IZBE). 


\section{Results}

\subsection{Description of New Species}

Docosia caucasica sp. n. (Figures 1 and 2).

LSID urn:lsid:zoobank.org:act: 6B1E8E5E-E745-44B4-A270-24973EF6C913.

Type material. Holotype. Male, GEORGIA, Samtskhe-Javakheti, road from Abastumani to Saime, near river, $41^{\circ} 46.63^{\prime} \mathrm{N}, 42^{\circ} 50.23^{\prime}$ E, $1370 \mathrm{~m}$ a.s.l. 10-11.vi.2019, Malaise trap, X. Mengual leg. (in ethyl alcohol, terminalia in glycerine, ZFMK-DIP-00082491; one fore leg used for DNA extraction, GenBank accession number: OL619794).

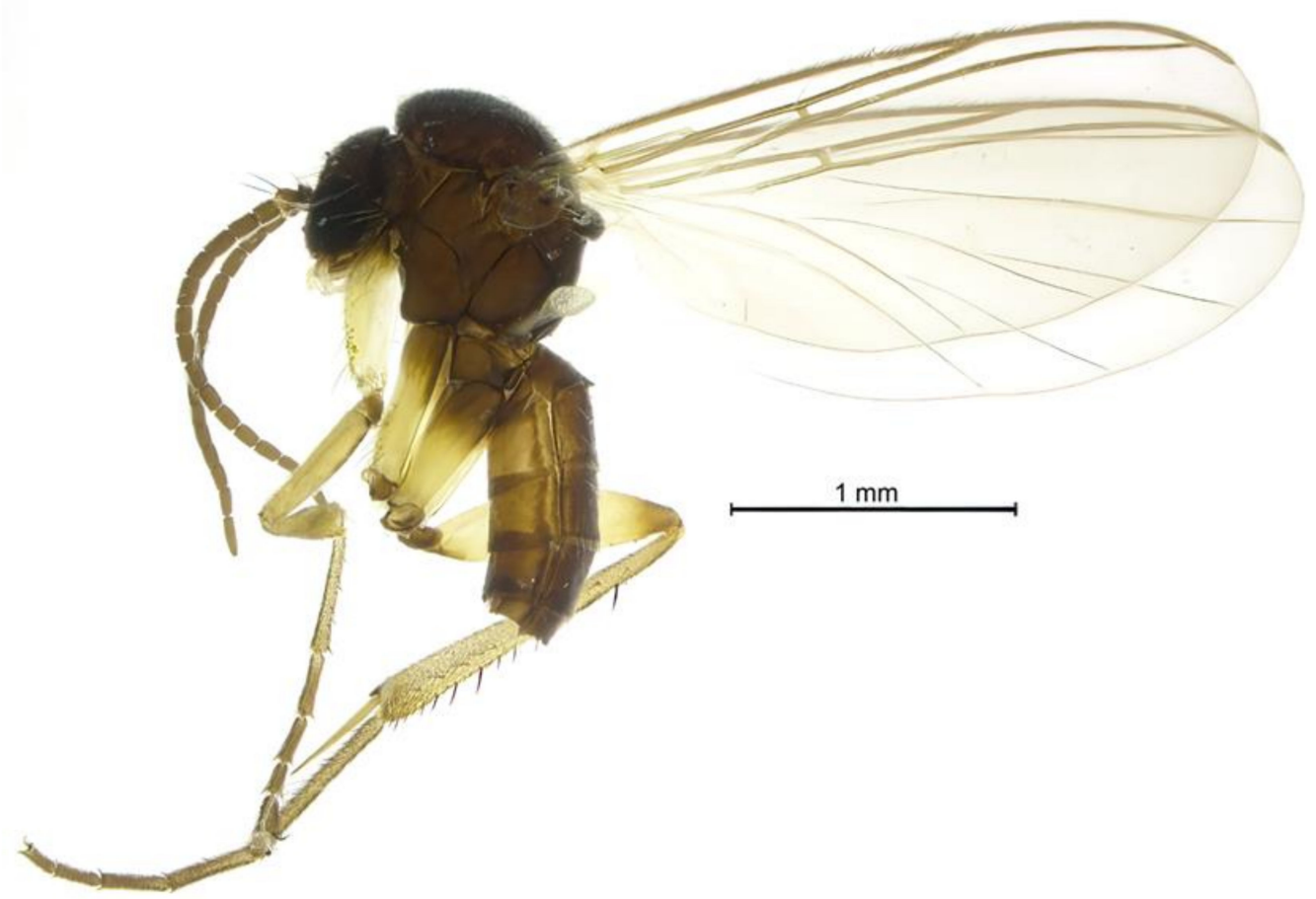

Figure 1. Docosia caucasica sp. n., o'(ZFMK-DIP-00082491), habitus, terminalia detached.

Differential diagnosis. Based on the structure of the male terminalia, D. caucasica sp. $\mathrm{n}$. belongs to a group of Palaearctic species that have posteroventral margin of the gonocoxites with (1) a prominent medial process of variable shape bearing dense aggregation of modified setae, and (2) distinct lateral processes. In this group, the new species resembles $D$. landrocki Laštovka and Ševčík, 2006 by having a similar outline of the medial process of the posteroventral margin of the gonocoxites and the gonostylus [8] (Figure 8), (Figure 3A-F). However, D. caucasica sp. $\mathrm{n}$. has (1) the posterolateral process of the gonocoxites ventrally tapering, extending to the posterior third of the gonostylus (posterolaterally drawn out to small process that bears an aggregation of medially directed long setae and an apical spine-like prolongation in $D$. landrocki), (2) dorsal lobe of the gonostylus posteromedially drawn out to a small extension (dorsal lobe of the gonostylus widening posteriorly, without an extension in D. landrocki), (3) ventral lobe of the gonostylus with posterior prong bearing two clearly delimited, apically pointed subequal spines (posterior prong with apically spathulate and basally fused two long subequal spines in D. landrocki), (4) ventral lobe of the gonostylus with anterior prong bearing an apical sabre-shaped spine (anterior prong apically bifid, with small spine apically on more posterior ramus in D. landrocki), (5) parameral apodeme in ventral view widened posteriorly (evenly rounded in D. landrocki), and (6) aedeagus with well elongated hook-like apical part (aedeagus hooked, not elongated in D. landrocki). 


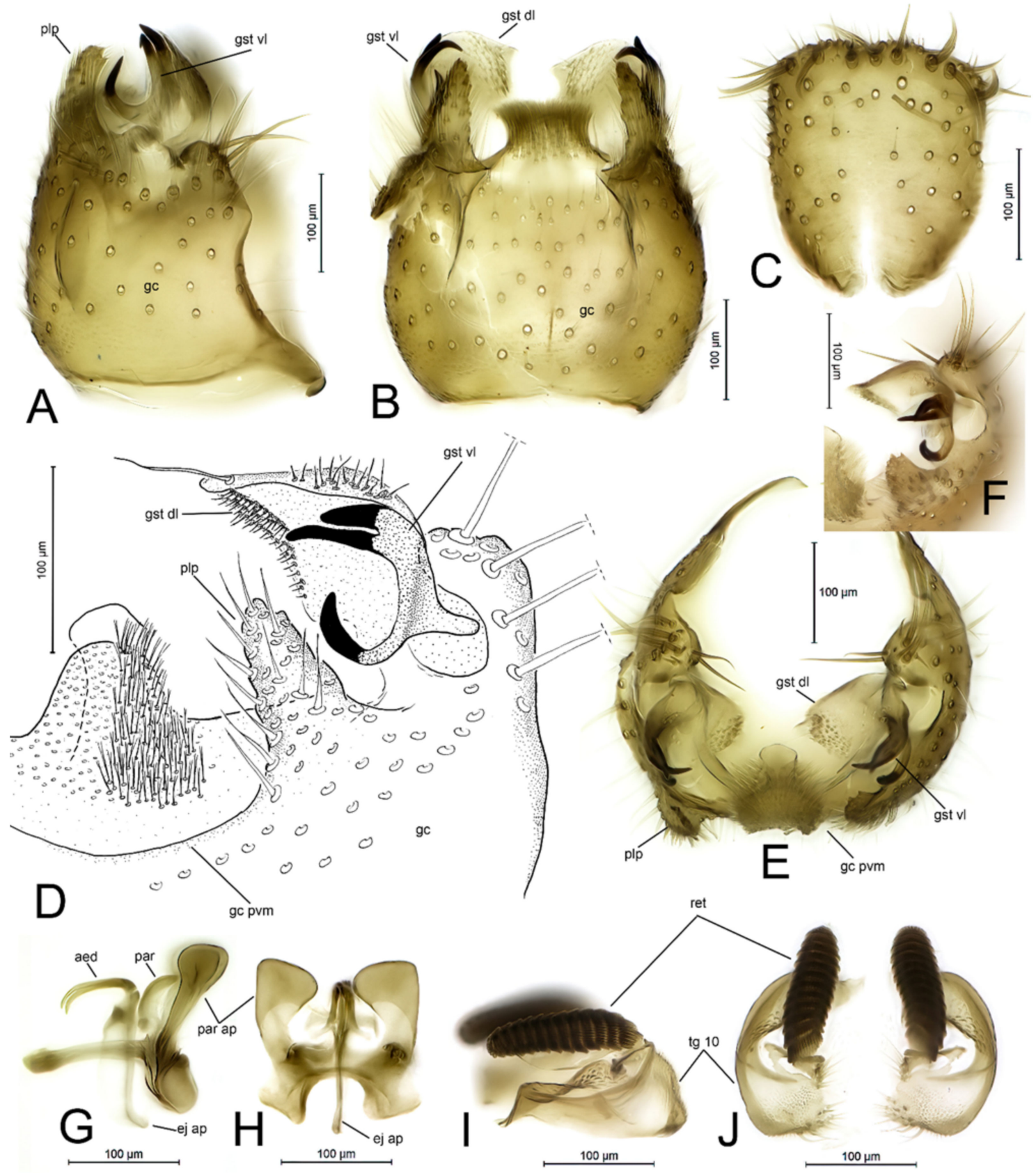

Figure 2. Docosia caucasica sp. n., ơ [ZFMK-DIP-00082491], terminalia. (A) Lateral view; (B) Ventral view; (C) Dorsal view of tergite 9; (D) Postero-ventral view of gonostylus and gonocoxite; (E) Posterior view; (F) Postero-ventral view of gonostylus; (G) Lateral view of aedeagal complex; (H) Ventral view of aedeagal complex; (I) Lateral view of cerci; (J) Dorsal view of cerci. Abbreviations: aed = aedeagus; ej ap = ejaculatory apodeme; gc = gonocoxite; gc pvm = posteroventral margin of gonocoxite; gst $\mathrm{vl}$ = ventral lobe of gonostylus; gst $\mathrm{dl}$ = dorsal lobe of gonostylus; par = paramere; par ap = parameral apodeme; $\mathrm{plp}=$ posterolateral process of gonocoxite; ret $=$ combs of retinacula.

Description. Male $(n=1)$. Length of wing $2.7 \mathrm{~mm}$; ratio of length to width 2.67 .

Head blackish brown with numerous pale setae. Three ocelli, the laterals separated from the eye margins by approximately their own diameter. Mouthparts light brownish. Palpus with two basal segments brownish and three apical segments yellow. Scape, pedicel and all flagellomeres dark brown. Flagellomeres cylindrical, flagellomeres 113 about 1.6 times as long as broad, apical flagellomere conical, about 3.8 times as long as broad basally. 
Thorax all dark brown to blackish but somewhat lighter than head, with light setae. Scutellum with numerous pale setae and several submarginal pale bristles about twice as long as scutellum. Antepronotum and proepisternum with pale bristles. Laterotergite and other pleural parts bare. Haltere pale yellow.

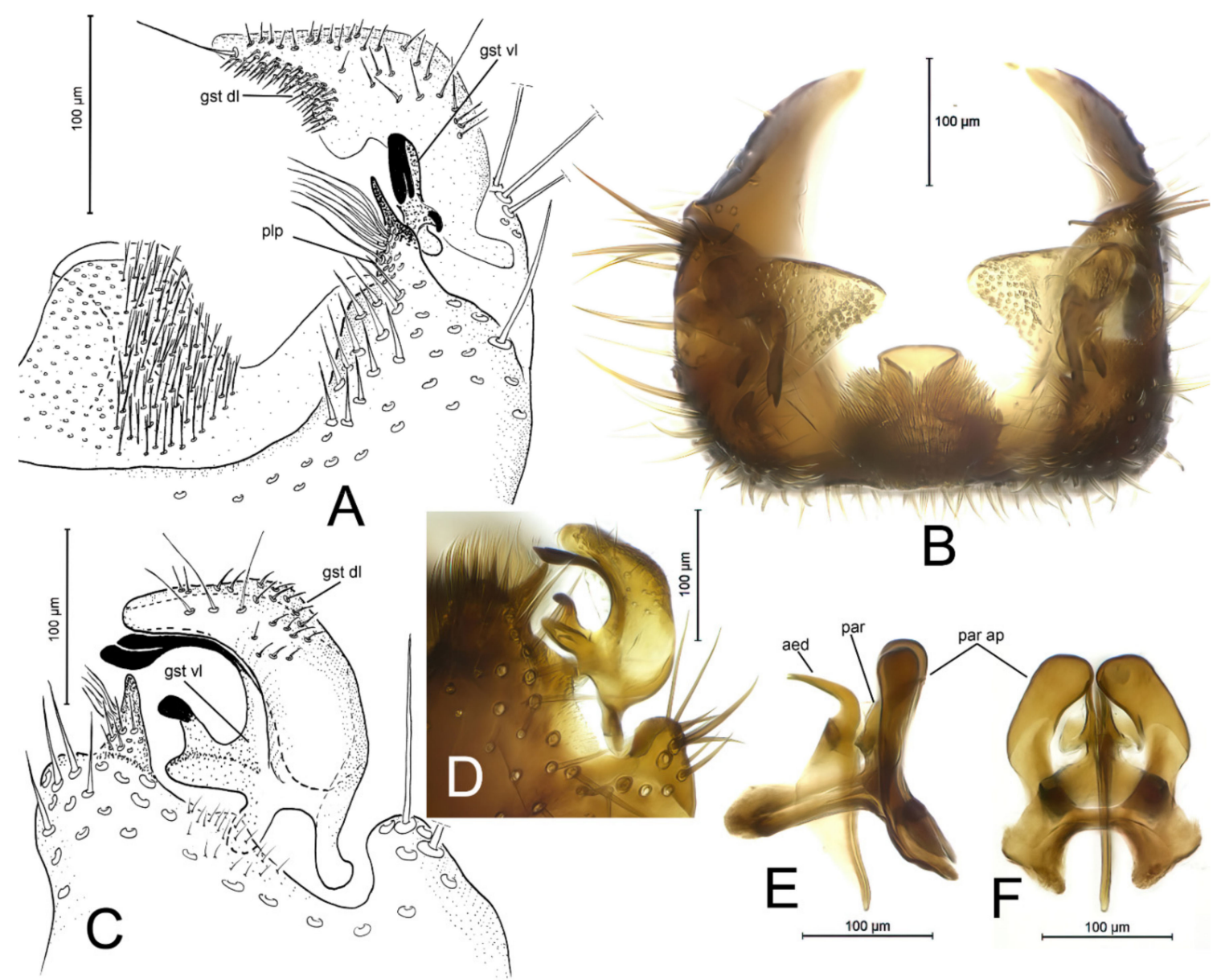

Figure 3. Docosia landrocki Laštovka and Ševčík, 2006, ơ(IZBE0222620), terminalia. (A) Postero-ventral view of gonostylus and gonocoxite; (B) Posterior view. (C,D) Lateral view of gonostylus. (E) Lateral view of aedeagal complex. (F) Ventral view of aedeagal complex. For abbreviations see Figure 2.

Legs. All coxae yellow, with basal third of mid- and hind coxae darkened. Trochanters brownish. Mid legs absent in holotype beyond trochanters. Fore- and hind femora mostly yellow, except hind femur apically darkened. Fore- and hind tibiae yellow. Fore tibia apicomedially with a semi-circular tibial organ (anteroapical depressed area), without strong setae, only densely covered with fine setulae. Tarsi seem darker because of dense setae.

Wing hyaline, unmarked. Radial veins and $\mathrm{r}-\mathrm{m}$ dark brown, other veins paler, $\mathrm{m}$-stem and basal parts of $\mathrm{M}_{1}$ and $\mathrm{M}_{2}$ faint, almost not traceable. Sc, Rs, bm-m, m-stem, basal part of $\mathrm{M}_{4}$ and basal half of cu-stem asetose, the other veins setose. Costa reaches to about the third of the distance between $R_{5}$ and $M_{1}$. Sc ends in $R$ before the level of beginning of $\mathrm{m}$-stem. Posterior fork begins before anterior fork, approximately at the middle of $\mathrm{r}-\mathrm{m}$.

Abdomen dark brown, with second and third segments lighter. Terminalia (Figure 2A-J) light brown. Tergite 9 basally rounded, with small medial incision, apically blunt, longer than broad, posterior margin slightly convex, setose with row of subapical stronger setae. Posteroventral margin of gonocoxites with extended flange; medial, somewhat tapering wide lobe densely covered with apically ramified setae. Dorsally from medial lobe and extending over it apically, spathulate, apically widened bare lobe, well discernible from posterior view. Laterally from medial lobe, posteroventral margin of gonocoxite drawn 
out to prominent posterolateral setose process extending to apical third of gonostylus. Gonostylus consists of two lobes: (1) large crescent-shaped dorsal lobe that is medially membranous, medially and laterally with short setae and with a long deviating subapical seta, and (2) ventral lobe that is split into three prongs, one medially directed anterior prong and two more posterior and posteriorly directed prongs, all with black apical spines. Parameral apodeme posteriorly widened and blunt. Adeagus with elongated hook-like apical part. Cercus with 13 combs of small spines (retinacula).

Female. Unknown.

Biology. Unknown.

Etymology. The species name refers to the Caucasian type locality.

\subsection{DNA Analysis}

DNA analysis was able to further confirm the morphological conclusions. The cytochrome c oxidase subunit 1 (COI) sequence of $D$. caucasica sp. n. was significantly different from other Docosia species found in GenBank. Further analysis revealed that while the newly described species was most similar to $D$. landrocki, with which it grouped together on $97 \%$ of bootstrap trees (Figure 4), genetic distance between the two was still considerable. In fact, the COI sequence of $D$. landrocki varied form that of D. caucasica sp. n. by $9.92 \%$ base differences per site (Table 1 ).

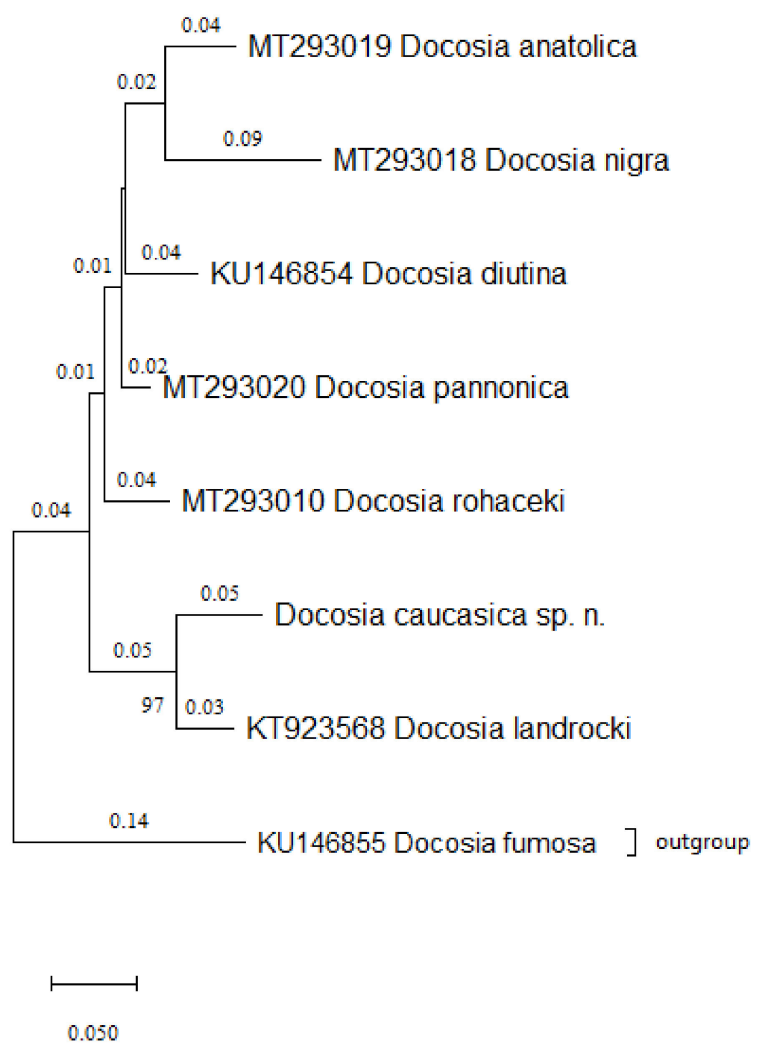

Figure 4. Maximum likelihood hypothesis for the relationships between a group of Docosia spp., constructed by using the General Time Reversible model [24]. A discrete Gamma distribution was used to model evolutionary rate differences among sites ( 5 categories $(+G$, parameter $=0.2193)$ ), and the tree is drawn to scale. 
Table 1. Table showing the pairwise Kimura 2-parameter distances between sequences [25]. Standard error estimates are shown above the diagonal.

\begin{tabular}{llcccccccc}
\hline & & $\mathbf{1 .}$ & $\mathbf{2 .}$ & $\mathbf{3 .}$ & $\mathbf{4 .}$ & $\mathbf{5 .}$ & $\mathbf{6 .}$ & $\mathbf{7 .}$ & $\mathbf{8 .}$ \\
\hline 1. & OL619794 D. caucasica sp. n. & & 0.024 & 0.030 & 0.036 & 0.034 & 0.046 & 0.049 & 0.052 \\
\hline 2. & KT923568 D. landrocki & 0.099 & & 0.035 & 0.028 & 0.038 & 0.033 & 0.050 & 0.045 \\
\hline 3. & MT293010 D. rohaceki & 0.140 & 0.153 & & 0.016 & 0.024 & 0.022 & 0.043 & 0.041 \\
\hline 4. & MT293020 D. pannonica & 0.156 & 0.124 & 0.068 & & 0.019 & 0.016 & 0.029 & 0.035 \\
\hline 5. & MT293019 D. anatolica & 0.154 & 0.179 & 0.116 & 0.083 & & 0.025 & 0.038 & 0.054 \\
\hline 6. & KU146854 D. diutina & 0.199 & 0.155 & 0.094 & 0.066 & 0.112 & & 0.044 & 0.043 \\
\hline 7. & MT293018 D. nigra & 0.208 & 0.216 & 0.192 & 0.135 & 0.165 & 0.190 & & 0.047 \\
\hline 8. & KU146855 D. fumosa (outgroup) & 0.246 & 0.216 & 0.205 & 0.171 & 0.261 & 0.209 & 0.223 \\
\hline
\end{tabular}

\section{Discussion}

In spite of more than two centuries of research history regarding Mycetophilidae in Europe that could have provided an exhaustive synopsis, the regional fauna still conceals a substantial amount of undescribed diversity (cf. [26]). However, in other regions of the world, Mycetophilidae are even more superficially studied and/or have been almost neglected for a long time, as is the case in Transcaucasia. The first checklist of Mycetophilidae in Georgia was published only recently, still representing probably less than half of the actual local diversity, which in turn undoubtedly includes a substantial number of undescribed species [27]. Therefore, discovery of a new species from the Lesser Caucasus in Georgia is unsurprising.

Docosia caucasica sp. n. was represented by a single specimen, which is relatively common as $33 \%$ of Georgian species are so far known from singletons [27]. Moreover, in the Malaise trap sample containing the holotype, seven out of the eight additional Mycetophilidae species were also represented by only a single specimen. This bias towards rarity can be explained by insufficient sampling, but as discussed by earlier authors, a substantial proportion of diversity tends to be rare [28,29]. Flather and Sieg [30] considered the majority of species in an ecological community to be represented by a few individuals only. As an example, Coddington et al. [31] described a high frequency of singletons $(=32 \%$ on average) in tropical arthropod surveys. In turn, Lim et al. [29] discussed that additional sampling is usually of limited help in decreasing the proportion of the singletons, because with supplementary material even more new singleton species are being collected. For communication purposes, whether inside and/or outside the scientific community, those rare species require naming. Consequently, a considerable proportion of new species have so far been described from only singletons, representing a common practise in entomology. According to Deng et al. [32], from about 4800 new insect species described in ZooKeys between 2009 and 2017, more than $20 \%$ were based on a single specimen. In the case of Mycetophilidae, 410 new species have been described during the last 20 years (2001-2020) in Zootaxa, the world's foremost journal of zootaxonomy [33], whereas 33\% of them are described from a singleton.

Indeed, according to the International Code of Zoological Nomenclature, there are no restrictions to describing new species from singletons until other requirements are met $[34,35]$. The new species must be unambiguously diagnosed in a way that ensures its identification when recollected. Moreover, deposited physical type material is also mandatory, as that allows re-examination and, if necessary, further testing of the scientific hypothesis regarding species delimitation [36]. In the case of Mycetophilidae, speciesspecific morphological characters are found mostly in male terminalia, whereas females are frequently determined only to the genus level or not included in a study at all. Therefore, a newly found Mycetophilidae species should be described with emphasize on the morphology of the male terminalia. However, several species have been historically de- 
scribed from a single female holotype, something that has generally been avoided during more recent decades (and should be abstained from in the future). In fact, female-based descriptions usually lead to many subsequent disadvantages, because the association of sexes in Mycetophilidae is frequently questionable and grounded in vague morphological argumentation. However, combining such information with DNA sequence data provides a far more solid basis for unambiguously determining which females and males belong to the same species (e.g. [37]).

The upturn of Docosia studies in Europe as well as in other regions has resulted in a number of descriptive and phylogeny based papers [7], forming a solid background for new species discovery and formal description. As shown by Fontaine et al. [38], a recent revision of the group has considerably shortened the time between species collecting and description that was otherwise approximately 21 years. In the case of Docosia, it has not been a single monographic revision but a set of papers, which have introduced proper diagnoses, including detailed figures of the male terminalia, for the majority of extant species. Moreover, for at least 30 of the species, genetic information in the form of most frequently used nuclear and mitochondrial markers is now publicly available [7]. Previous studies of Palaearctic Docosia species (e.g. $[7,9,12,13])$ show that the diagnostic characters of male terminalia are conservative, allowing for species identification based on minimal material. Taking into account the rather well described and illustrated assemblage of Docosia species as well as the extensive material studied earlier, we are convinced of having delimited a new species-Docosia caucasica sp. n.

Based on a combined analysis of five DNA markers, Ševčík et al. [7] discussed intrageneric phylogeny of Docosia with distinguishing a group of species having also a well-defined morphology of the male terminalia: longitudinal tergite 9, distinct posterolateral processes of gonocoxites and apically modified setae at posteroventral margin of the gonocoxites medially. In addition to six species, namely D. nigra, 1928, D. landrocki, D. anatolica, D. diutina, D. rohaceki and D. pannonica, included in the group by Ševčík et al. [7], several other species appear to belong here as well, namely D. helveola Chandler, 2004, D. turkmenica Zaitzev, 2011, D. blagoderovi Kurina and Ševčík, 2012, D. distributa Kurina and Ševčík, 2012 and D. trispinosa Kurina and Ševčík, 2013. Within the group, D. caucasica sp. n. is most similar to D. landrocki, distinguished by characters as shown in Diagnosis above.

It should be noted, that D. caucasica sp. n. proved to be distinct from other Docosia species based on the commonly used genetic marker cytochrome c oxidase subunit 1 (COI). This was in accordance with the morphological finds, especially as based on further analysis, $D$. landrocki was indeed the closest known relative to the new species. DNA barcoding based on mitochondrial sequences alone, especially when using universal primers, may lead to erroneous conclusions [39]. However, in this case the COI sequence of D. caucasica sp. $\mathrm{n}$. fit well into the narrative created by previous phylogenetic studies on Docosia [7]. Indeed, according to analysis, the genetic distance between $D$. caucasica sp. n. and D. landrocki is comparable to the differences among other known species of this genus (Table 1).

To conclude, in the aforementioned circumstances we consider it better to describe a new species from a single male specimen and keep the wider audience informed, rather than store the material in a drawer and wait decades for further data. In addition, we have not described the new species in isolation, something that is discouraged by multiple taxonomic journals. Instead, it was compared to information from a large set of recent taxonomy papers on Palaearctic Docosia and discussed in that context. Nevertheless, highquality standards in a new species delimitation must be implemented and there cannot be any trade-off on the basis of insufficient material.

Author Contributions: Conceptualization, O.K.; methodology, O.K. and H.K.; validation, O.K. and H.K.; investigation, O.K. and H.K.; resources, O.K.; data curation, O.K. and H.K.; writing-original draft preparation, O.K. and H.K.; writing-review and editing, O.K. and H.K.; visualization, O.K. and H.K.; supervision, O.K.; project administration, O.K.; funding acquisition, O.K. All authors have read and agreed to the published version of the manuscript. 
Funding: The study visit of O.K. to the Zoological Research Museum Alexander Koenig, Bonn, Germany was funded by the European Commission's Research Infrastructure program SYNTHESYS+ (DE-TAF-2498).

Institutional Review Board Statement: Not applicable.

Informed Consent Statement: Not applicable.

Data Availability Statement: All data is available in this paper.

Acknowledgments: We are grateful to X. Mengual (Bonn, Germany), for providing the specimen for this study and for information on collecting locality in Georgia. We would like to express our thanks to three anonymous reviewers for their valuable comments and suggestions on this manuscript.

Conflicts of Interest: The authors declare no conflict of interest. The funders had no role in the design of the study; in the collection, analyses, or interpretation of data; in the writing of the manuscript, or in the decision to publish the results.

\section{References}

1. Edwards, F. British Fungus-Gnats (Diptera, Mycetophilidae). With A Revised Generic Classification of the Family. Trans. R. Entomol. Soc. Lond. 1925, 73, 505-670. [CrossRef]

2. Hutson, A.M.; Ackland, D.M.; Kidd, L.N. Mycetophilidae (Bolitophilinae, Ditomyiinae, Diadocidiinae, Keroplatinae, Sciophilinae and Manotinae) Diptera, Nematocera. In Handbooks for the Identification of British Insects; Royal Entomological Society: London, UK, 1980; Volume 9, pp. 1-111.

3. Zaitzev, A.I. Fungus Gnats of the Fauna of Russia and Adjacent Regions. Part 1; Nauka: Moscow, Russia, 1994; 288p. (In Russian)

4. Ševčík, J.; Kaspřák, D.; Tóthová, A. Molecular phylogeny of fungus gnats (Diptera: Mycetophilidae) revisited: Position of Manotinae, Metanepsiini, and other enigmatic taxa as inferred from multigene analysis. Syst. Entomol. 2013, 38, 654-660. [CrossRef]

5. Kaspřák, D.; Kerr, P.; Sýkora, V.; Tóthová, A.; Ševčík, J. Molecular phylogeny of the fungus gnat subfamilies Gnoristinae and Mycomyinae, and their position within Mycetophilidae (Diptera). Syst. Entomol. 2019, 44, 128-138. [CrossRef]

6. Oliveira, S.S.; Amorim, D.S. Phylogeny, classification, Mesozoic fossils, and biogeography of the Leiinae (Diptera: Mycetophilidae). Bull. Am. Mus. Nat. Hist. 2021, 446, 1-108. Available online: http:/ / digitallibrary.amnh.org/handle/2246/7256 (accessed on 10 October 2021). [CrossRef]

7. Ševčík, J.; Burdíková, N.; Kaspřák, D.; Kurina, O. Five new Palaearctic species of Docosia (Diptera: Mycetophilidae), with updated molecular phylogeny of the genus. Eur. J. Taxon. 2020, 717, 3-26. [CrossRef]

8. Laštovka, P.; Ševčík, J. A review of the Czech and Slovak species of Docosia Winnertz (Diptera: Mycetophilidae), with atlas of the male and female terminalia. Čas. Slez. Muz. Opava A 2006, 55, 1-37.

9. Kurina, O. Three new species of Docosia Winnertz (Diptera: Mycetophilidae) from Kazakhstan. Entomol. Fennica 2006, 17, 110-117. [CrossRef]

10. Ševčík, J.; Kaspřák, D.; Rulik, B. A new species of Docosia Winnertz from Central Europe, with DNA barcoding based on four gene markers (Diptera, Mycetophilidae). ZooKeys 2016, 549, 127-143. [CrossRef]

11. Zaitzev, A.I. Two new species of the genus Docosia Winnertz (Diptera: Mycetophilidae) from Russia and Turkmenistan. Russ. Entomol. J. 2011, 20, 207-209. [CrossRef]

12. Kurina, O.; Ševčík, J. Three new species of Docosia Winnertz from central and southern Europe (Diptera: Mycetophilidae). Zootaxa 2011, 2810, 26-36. [CrossRef]

13. Kurina, O.; Ševčík, J. Notes on Docosia Winnertz (Diptera: Mycetophilidae), with description of six new species from Central Asia and the first generic record from the Afrotropical region. Zootaxa 2012, 3570, 25-40. [CrossRef]

14. Taber, S.W. A new Nearctic species of Docosia Winnertz fungus gnat (Diptera: Mycetophilidae), notes on Docosia walpurga Taber and Docosia dichroa Loew, and the identification of females of all three species. Southwest Entomol. 2012, 37, 379-390. [CrossRef]

15. Taber, S.W. A new Nearctic species of Docosia Winnertz fungus gnat (Diptera: Mycetophilidae) from Michigan. Southwest. Entomol. 2018, 43, 683-689. [CrossRef]

16. Ševčík, J. Docosia heikkii sp. nov., the fi rst Oriental record of Docosia (Diptera: Mycetophilidae). Orient. Insects 2010, 44, 91-94. [CrossRef]

17. Oliveira, S.S.; Amorim, D.S. Docosia adusta sp. nov. (Diptera, Mycetophilidae) from the Colombian Andes: A Holarctic element in northwestern South America. Can. Entomol. 2011, 143, 688-696. [CrossRef]

18. Oliveira, S.S.; Amorim, D.S. Catalogue of Neotropical Diptera. Mycetophilidae. Neotrop. Dipt. 2014, 25, 1-87.

19. Søli, G.E.E. Mycetophilidae (Fungus Gnats). In Manual of Afrotropical Diptera; Nematocera \& Lower, Brachycera; Kirk-Spriggs, A.H., Sinclair, B.J., Eds.; South African National Biodiversity Institute: Pretoria, South Africa, 2017; Volume 2, pp. 533-555.

20. Folmer, O.; Black, M.; Hoeh, W.; Lutz, R.; Vrijenhoek, R. DNA primers for amplification of mitochondrial cytochrome c oxidase subunit I from diverse metazoan invertebrates. Mol. Mar. Biol. Biotechnol. 1994, 3, 294-299. 
21. Hall, T.A. BioEdit: A user-friendly biological sequence alignment editor and analysis program for Windows 95/98/NT. Nucleic Acids Symp. Ser. 1999, 41, 95-98.

22. Tamura, K.; Stecher, G.; Kumar, S. MEGA 11: Molecular Evolutionary Genetics Analysis Version 11. Mol. Biol. Evol. 2021, 38, 3022-3027. [CrossRef]

23. Kurina, O. Cluzobra matilei sp.n. from French Guyana, with notes on congeners (Diptera: Mycetophilidae). Zootaxa 2008, 1874, 63-68. [CrossRef]

24. Nei, M.; Kumar, S. Molecular Evolution and Phylogenetics; Oxford University Press: New York, NY, USA, 2000; 348p.

25. Kimura, M. A simple method for estimating evolutionary rate of base substitutions through comparative studies of nucleotide sequences. J. Mol. Evol. 1980, 16, 111-120. [CrossRef]

26. Kjærandsen, J.; Søli, G.E.E. Updated checklist of Norwegian Mycetophilidae (Diptera) with 92\% DNA barcode reference coverage. Norw. J. Entomol. 2020, 67, 201-234.

27. Kurina, O. A contribution towards checklist of fungus gnats (Diptera, Diadocidiidae, Ditomyiidae, Bolitophilidae, Keroplatidae, Mycetophilidae) in Georgia, Transcaucasia. ZooKeys 2021, 1026, 69-142. [CrossRef] [PubMed]

28. Papp, L. "Outlaws": Some evolutionary aspects of rarity in insects. Tiscia 1998, 31, 29-33.

29. Lim, G.S.; Balke, M.; Meier, R. Determining Species Boundaries in a World Full of Rarity: Singletons, Species Delimitation Methods. Syst. Biol. 2012, 61, 165-169. [CrossRef] [PubMed]

30. Flather, C.H.; Sieg, C.H. Species rarity: Definition, causes, and classification. In Conservation of Rare or Little-Known Species: Biological, Social, and Economic Considerations; Raphael, M.G., Molina, R., Eds.; Island Press: Washington, DC, USA, 2007; pp. 44-66.

31. Coddington, J.A.; Agnarsson, I.; Miller, J.A.; Kunter, M.; Hormiga, G. Undersampling bias: The null hypothesis for singleton species in tropical arthropod surveys. J. Anim. Ecol. 2009, 78, 573-584. [CrossRef] [PubMed]

32. Deng, J.; Guo, Y.; Cheng, Z.; Lu, C.; Huang, X. The Prevalence of Single-Specimen/Locality Species in Insect Taxonomy: An Empirical Analysis. Diversity 2019, 11, 106. [CrossRef]

33. Whitmore, D.; Gaimari, S.D.; Nihei, S.S.; Evenhuis, N.L.; Kurina, O.; Borkent, C.J.; Sinclair, B.J.; O’Hara, J.E.; Zhang, Z.-Q.; Moulton, J.K.; et al. Twenty years of Dipterology through the pages of Zootaxa. Zootaxa 2021, 4979, 166-189. [CrossRef]

34. International Code of Zoological Nomenclature. 4th Edition. Available online: http:/ /iczn.org/iczn/index.jsp (accessed on 20 October 2021).

35. Wells, A.; Johanson, K.A.; Dostine, P. Why are so many species based on a single specimen? Zoosymposia 2019, 14, 32-38. [CrossRef]

36. Amorim, D.S.; Santos, C.M.D.; Krell, F.-T.; Dubois, A.; Nihei, S.S.; Oliveira, O.M.P.; Pont, A.; Song, H.; Verdade, V.K.; Fachin, D.A.; et al. Timeless standards for species delimitation. Zootaxa 2016, 4137, 121-128. [CrossRef]

37. Kurina, O.; Õunap, E.; Ramel, G. Baeopterogyna mihalyii Matile (Diptera, Mycetophilidae): Association of sexes using morphological and molecular approaches with the first description of females. ZooKeys 2011, 114, 15-27. [CrossRef] [PubMed]

38. Fontaine, B.; Perrard, A.; Bouchet, P. 21 years of shelf life between discovery and description of new species. Curr. Biol. 2012, 22, R943-R944. [CrossRef] [PubMed]

39. Song, H.; Buhay, J.E.; Whiting, M.; Crandall, K.A. Many species in one: DNA barcoding overestimates the number of species when nuclear mitochondrial pseudogenes are coamplified. Proc. Natl. Acad. Sci. USA 2008, 105, 13486-13491. [CrossRef] [PubMed] 